\title{
ANÁLISIS DEL PROCESO DE DIFUSIÓN ESPACIAL DEL FÚTBOL PROFESIONAL EN CHILE
}

\author{
Pablo Barra Vieira ${ }^{1}$ y Francisco Maturana Miranda ${ }^{2}$ \\ Universidad de Concepción y Universidad Autónoma de Chile
}

\begin{abstract}
RESUMEN
El fútbol es considerado el deporte más popular a escala mundial, y en su modalidad profesional es capaz de generar entusiasmo y sentimientos de progreso en cada ciudad que forma parte. Pero ¿cómo se propaga esta actividad en el espacio? En el presente artículo, se analiza el proceso de difusión espacial del fútbol profesional en Chile, utilizando los criterios de propagación más comunes: por proximidad geográfica y jerarquía urbana. Los datos se obtuvieron a partir del año de adopción por parte de las ciudades, su población en ese instante, su localización geográfica y su distancia con respecto a la ciudad emisora. A través del tiempo, el fútbol profesional chileno se ha propagado espacialmente mediante un patrón guiado por la distancia y la cercanía al foco emisor, producto del poder institucional reflejado en la Asociación Central de Fútbol (ACF), el ente que decidía cuáles eran las ciudades que ingresaban a la actividad profesional, hasta inicios de la década de los años ochenta.
\end{abstract}

Palabras clave: Difusión espacial; fútbol profesional; Chile.

\section{ANALYSIS OF THE SPATIAL DIFFUSION PROCESS OF PROFESSIONAL FOOTBALL IN CHILE}

\section{ABSTRACT}

Football is considered the most popular sport worldwide, and in his professional mode is able to generate enthusiasm and a sense of achievement in each city that is part of it. But, by what process it was spread spatially over time? In this article the process of geographical spread of professional football in Chile is analyzed, following the most common propagation criteria: by geographical proximity and by urban hierarchy. Data were obtained from the year of adoption by cities, its population at the time, its geographical location and its distance from the main city. Over time, the Chilean professional football has spread spatially by various processes, following a pattern guided by the distance and proximity to the emitting source, a product of institutional power reflected in the Central Football Association (CFA), the entity that decided which cities entered the professional football, until the beginning of the 1980s.

Keywords: Spatial diffusion; professional football; Chile.

Fecha de recepción: 24 de marzo de 2014. Fecha de aceptación: 6 de mayo de 2015.

${ }^{1}$ Geógrafo, Universidad de Concepción.

${ }^{2}$ Investigador, Instituto Chileno de Estudios Municipales, Universidad Autónoma de Chile. 


\section{INTRODUCCIÓN}

El fútbol es uno de los deportes más populares a escala mundial. Esta actividad ha sido capaz de generar y movilizar grandes sumas de dinero, convirtiéndose en unos de los negocios más rentables del planeta. Lo anterior, se debe al importante ingreso que se percibe por las ventas de entradas, mercancías y por las ganancias derivadas de patrocinios y derechos de transmisión televisivos y radiales (DRUT, 2014; PACHÉ Y N'GOALA, 2011; OMAÑA, 2007).

Este deporte, nacido en 1863 en Inglaterra, momento en que se distanció del rugby y donde la Universidad de Cambridge intentó homogeneizar las reglas (FIFA, 2014a), sufrió una rápida expansión. Hacia 1888 se preparaba un campeonato donde existían más de 50 clubes en dicho País. Su difusión en otras latitudes se debió -principalmente- a la influencia inglesa en otros países como: Holanda y Dinamarca (1889); Argentina (1893) y Chile, (1895) entre otros. El punto culmen de este primer momento significó la fundación en 1904 de la Fédération Internationale de Football Association (FIFA), en la ciudad de París (Francia).

El fútbol, como toda actividad, está vinculado al espacio y territorio. En este sentido, su carácter universal, el dominio cultural que produce en los diferentes lugares, lo hace transformarse en un deporte de suma importancia en términos sociales, culturales y económicos (RAVENEL, 1997).

Así, la distribución espacial del fútbol profesional, no escapa a la concentración de población que reside en centros urbanos y la capacidad que tienen estos en sustentar la actividad (RAVENEL, 1998). En efecto, la importancia de los equipos estaría vinculada a su capacidad de poseer un capital humano para su administración, espectadores y volumen económico de todos los elementos que puedan implicar una actividad deportiva (MEYSSONNIER Y MINCHENEAU, 2013), lo cual en cierta medida ocasiona que los equipos de fútbol sean un excelente indicador de la jerarquía o plaza que ocupan las ciudades en un sistema urbano, esto último dada la correlación existente entre esta y la importancia de los equipos (THÉRY, 2006).

Bajo la misma lógica anterior, la globalización y las mejoras en las comunicaciones generaron que los clubes de futbol no solo tuvieran un papel relevante en términos de campeonato local, sino cada vez más internacionalmente con múltiples competiciones que incluso escapan a la escala continental (SCHOTTÉ, 2014).

Desde un punto de vista territorial, el fútbol se ha estudiado como un elemento que estructura las sociedades y los territorios. El interés por este deporte se ha plasmado en una respetable cantidad de estudios (BALE, 1978; BOUKERZAZA, 2006; CAPEL, 2001; MASCARENHAS, 2008; RAVENEL, 1997). Bajo esta lógica espacial, los conceptos de fútbol y difusión espacial se entrecruzan. Esto asociado a la forma de adopción de la actividad en las ciudades, su relación con la jerarquía urbana presente y los procesos socio-económicos que involucraron el nacimiento de la actividad. Ello ha quedado expresado en diversos trabajos asociados al potencial demográfico, a la modernidad, a las consideraciones teóricas y metodológicas, a los procesos de difusión, al paisaje y a la globalización e identidad nacional que genera esta actividad (BALE, 1998; GROSJEAN, 2006; MASCARENHAS, 1998, $2000 \mathrm{Y}$ VILLENA, 2003).

Por lo tanto, existirán diferentes procesos y factores que intervienen directamente en cómo surge la actividad en las diferentes ciudades y donde su difusión espacial toma relevancia, al identificar y comprender cómo un fenómeno se propaga en el ambiente, involucrando variables de espacio y tiempo (PICKENHAYN, 2001). Este concepto de difusión espacial emerge gracias a las contribuciones de Hägerstrand (1966) a partir de sus primeros estudios aplicados a las innovaciones en el distrito agrícola de Asby, Suecia. El proceso, según Pumain y Saint-Julien (2001) se produce a partir de un lugar emisor, es decir, desde un lugar que da el impulso y "posibilita la propagación y donde su fuerza proviene de la reunión de actores iniciadores del movimiento. Esta fuerza inicial es determinante para la transmisión de una idea, puesto que en un proceso de difusión espacial, todos los lugares no tienen la misma vocación a ser un lugar emisor" (PUMAIN Y SAINT-JULIEN, 2001, P. 171). 
Brown (1968, EN INFANTE, 1980) identificó seis elementos básicos comunes a todo proceso de difusión: (1) un área, (2) una dimensión temporal, (3) una "innovación" que se difunde, (4) lugares en donde esa "innovación" está localizada al comienzo del intervalo de tiempo estudiado, (5) lugares en donde esa "innovación" está nuevamente localizada al término del intervalo de tiempo estudiado y (6) vías de movimiento, influencia y relaciones entre lugares de origen y destino de la innovación.

La teoría de la difusión considera que una vez que aparece una innovación al interior de un territorio, esta se puede difundir de dos formas: por cercanía, la cual como su nombre lo indica, consiste en una propagación que comienza primero en los sectores más próximos y posteriormente, en los más lejanos; y la segunda, se realiza de forma jerárquica, se propaga según el número de habitantes en cada territorio o la importancia de los receptores, introduciéndose primero en las áreas más pobladas, cuando nos expresamos en términos de ciudad (HÄNGERSTRAND, 1967, EN CAPO, 2007).

Así, el fútbol como toda innovación, tendrá ciertas particularidades en su aparición y propagación según el contexto social, económico, cultural y urbano de cada país. Particularmente en Chile, su aparición hacia fines del siglo XIX estará consolidada hacia 1926 con la unificación del fútbol nacional entre Asociación y Federación de este deporte (LOS SPORTS, 1926), comenzando así el primer campeonato profesional hacia 1933.

Bajo este escenario, en Chile, la existencia de trabajos que traten sobre fútbol y su propagación será casi nula. Sin embargo, es posible encontrar investigaciones que abordan este deporte desde otras perspectivas vinculados a la disciplinas de la sociología, historia, psicología y economía (BONNASSIOLLE, 2012; CASSASUS Y GÓMEZ, 2012; DURÁN, 2005 et al.; ESCOBAR, 2011; FIGALLO, MUÑOZ Y SALHE, 2003; GUERRERO 2006; MARAMBIO, 2010; PARRA, 2007; VALENZUELA Y VERGARA, 2013).

En este contexto y con los argumentos planteados, esta investigación buscó explorar y comprender la manera en que la actividad del fútbol profesional en Chile se propagó por el territorio. Se observó que las ciudades más próximas al foco emisor, asimilaron la innovación del fútbol profesional más tempranamente, dejando a un segundo plano (regularmente, pero no siempre) la cantidad de población para captar la actividad. No obstante, algunas ciudades de los extremos del territorio chileno, pese a su papel como capital regional, desarrollaron esta innovación más tardíamente, lo anterior asociado a los costos de desplazamiento.

Además, se observó que la propagación se presentó de manera más enérgica, a partir de1950 en línea con los primeros pasos de industrialización efectuados en el País, lo cual indicaría que dicho proceso fue el verdadero motor del desarrollo de la actividad.

\section{MÉTODO}

La metodología de esta investigación se desarrolló a partir de tres puntos. El primero consistió en delimitar los clubes de fútbol profesional en Chile. Para tal efecto, se seleccionaron a todos los equipos que han participado por lo menos en una temporada en alguno de los tres campeonatos organizados por la Asociación Nacional de Fútbol Profesional (ANFP). En la actualidad: Primera División, Primera B y Segunda División Profesional. Esta información fue recabada desde el sitio web de la Rec. Sport. Soccer Statistics Foundation ${ }^{3}$ y complementada con una revisión de los sitios oficiales de diferentes equipos y literatura especializada. Las ciudades que estaban compuestas por clubes son posibles de observar en la Figura 1.

\footnotetext{
${ }^{3}$ http://www.rsssf.com
} 
FIGURA 1

Ciudades con presencia de fútbol profesional*.

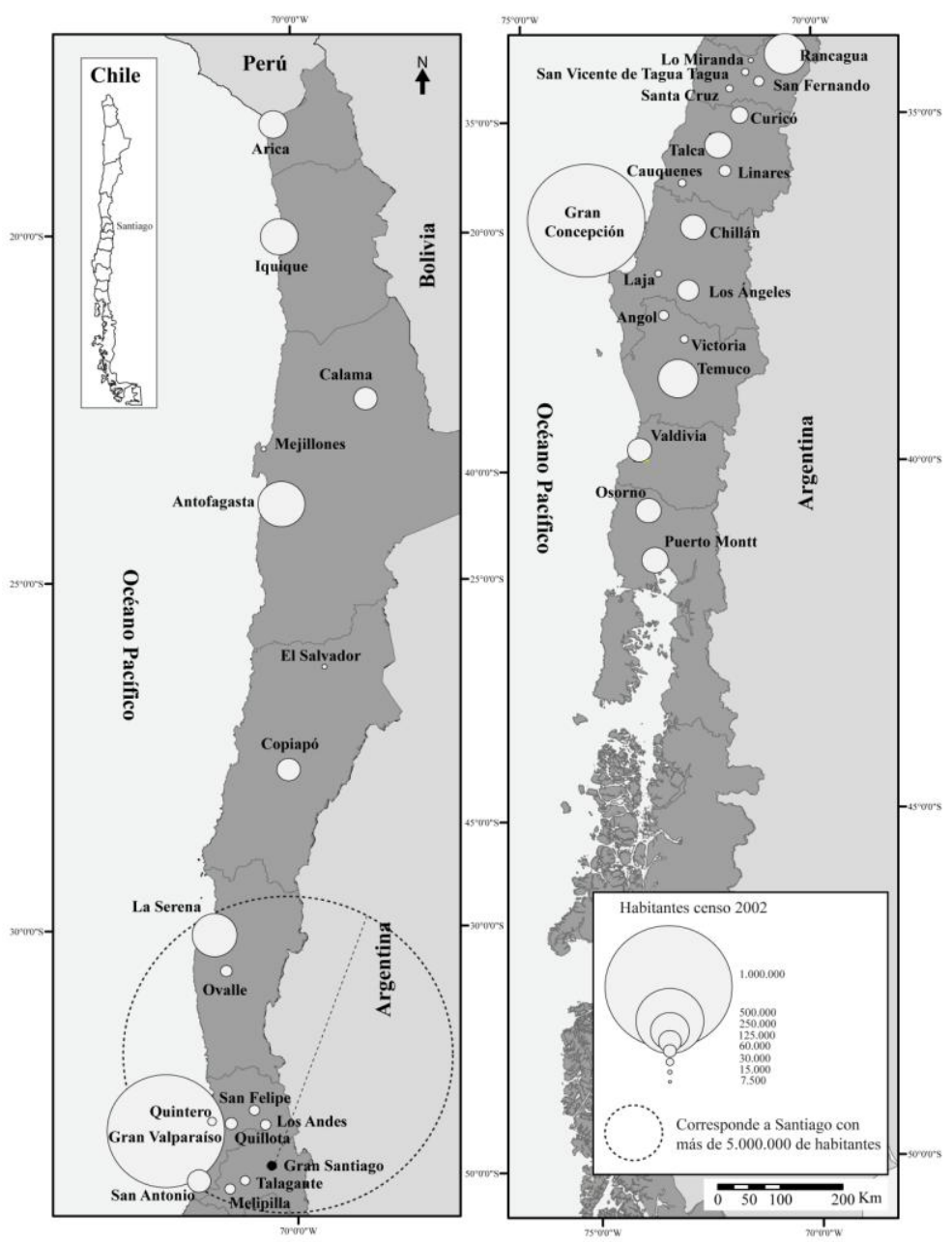

* Por motivos de solución gráfica Chile fue divido en 2 partes.

Fuente: elaboración propia a partir de INE, 2005.

Como segundo punto, se analizaron las fases del proceso de difusión espacial con el apoyo de tres gráficos. El primero estableció los años (en intervalo de 10), de la frecuencia acumulada $(\%)$ de los equipos de fútbol y las ciudades chilenas que habían adoptado el fútbol profesional en sus 81 años de existencia. Una segunda figura, fue confeccionada para comprender la variable tiempo y distancia (en kilómetros, desde el foco emisor de esta innovación), estas últimas obtenidas gracias al Ministerio de Obras Públicas de Chile ${ }^{4}$. Finalmente respecto a la difusión jerárquica, se elaboró un gráfico que expresa la relación entre el tamaño de población en las ciudades y el año en que se adoptó el fútbol profesional en cada una de ellas. Para tal efecto, se utilizó la cantidad de habitantes de cada ciudad, según el año de adopción. Dicho dato se extrajo del censo más cercano a la fecha de la aparición del fútbol en el centro urbano. Se utilizó, por tanto, el trabajo desarrollado por el Instituto Nacional de Estadísticas (INE, 1999) que permitió observar la población de los centros poblados en Chile desde 1875 al año 1992 y el censo del año 2002, publicado por el INE (2005) para observar la población de dicho año.

Todo lo anterior se complementó de manera transversal con una recopilación de textos que sustentaban los procesos vividos por el País en los diferentes periodos.

\footnotetext{
${ }^{4}$ http://servicios.vialidad.cl/Distancias/Distancias.asp, consultada el 2 de diciembre, 2014.
} 


\section{RESULTADOS Y DISCUSIÓN}

En la Figura 2 es posible observar el año de adopción y la frecuencia acumulada, lo que entrega un panorama de la concentración en la difusión de la actividad profesional del fútbol en Chile. En efecto, desde 1933 hasta el año 1953 se observa que seis ciudades adoptaron la actividad, las que corresponden al $16,2 \%$ del total, por lo que estas son consideradas "adoptadores tempranos", durante el inicio del proceso de difusión espacial. La primera ciudad que integró el profesionalismo, además del Gran Santiago (foco emisor), fue el Gran Valparaíso (a 115,95 km del centro difusor y donde arribó la actividad por parte de los ingleses) en 1937, cuando se incorporó S. Wanderers. Posteriormente, el proceso de industrialización, impulsado por el Estado en 1939, generó un crecimiento que se manifestó en la modernización y aumento de los medios de transporte, lo que es considerado como un elemento fundamental para que el fútbol profesional se expandiera desde Santiago hasta llegar a abarcar casi todo el territorio nacional (SANTA CRUZ, 1991).

A partir del año 1953, la consolidación de la Segunda División (creada en 1952) permitirá una incorporación importante de equipos vinculados a las ciudades de Rancagua, Talca, Curicó, Los Andes y otros clubes localizados en la Capital del País o en sus proximidades en lo que hoy en día es posible observar como la Región Metropolitana (ver figura 1). En este momento hacia 1956, será Dep. La Serena localizado en la ciudad del mismo nombre, uno de los primeros clubes más alejados del foco emisor (470 km aprox.) en sumarse al fútbol profesional.

El mundial del año 1962, realizado en Chile, sin duda, contribuyó en la difusión de este deporte. Cabe precisar, que durante ese mismo año, el campeonato de Primera División tuvo una cifra record de espectadores superando los 2.634 .538 personas (SANTA CRUZ, 1991). Este fenómeno, seguramente impactó en la difusión de la actividad y que se vio reflejado en que al año siguiente el centro urbano de Temuco, (ciudad localizada a $670 \mathrm{~km}$ aproximadamente del foco emisor (ver Figuras 1 y 3), se incorporara al fútbol profesional.

FIGURA 2

Curva de adopción del fútbol profesional en Chile.

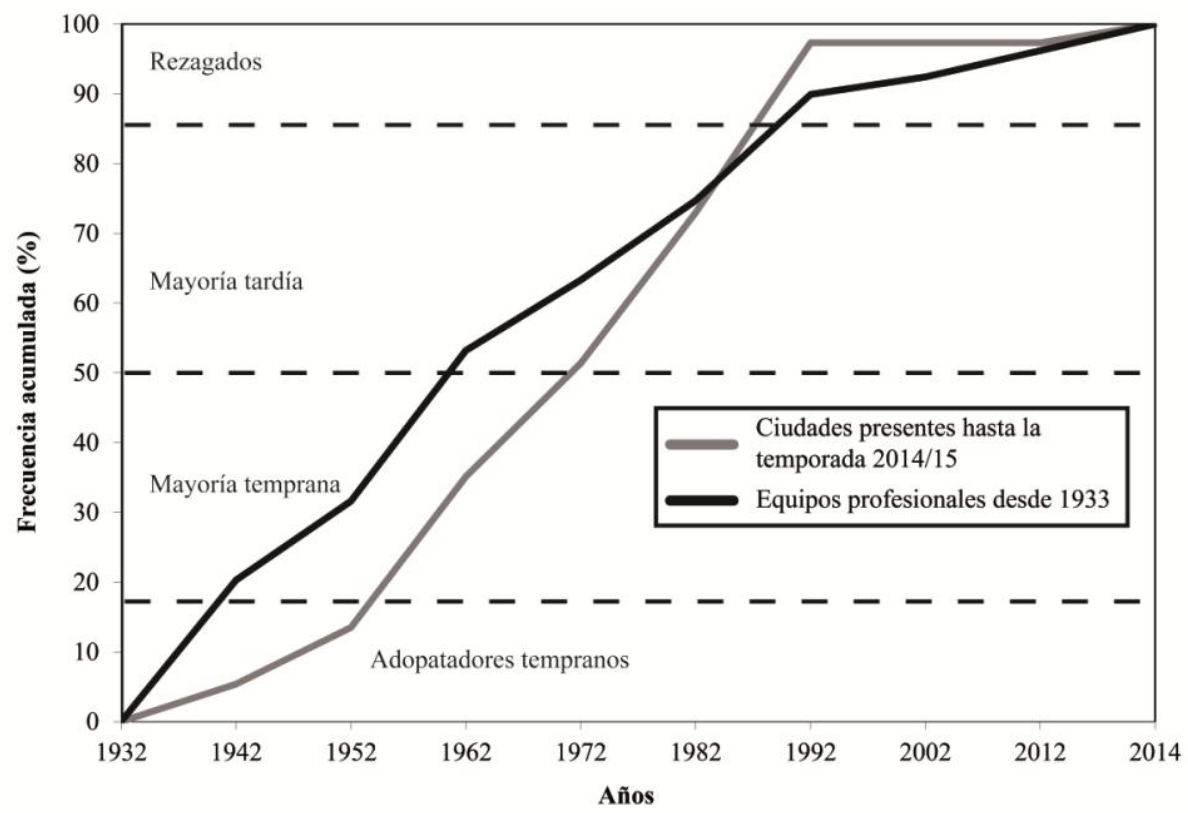

Fuente: Elaboración propia.

Otros aspectos a destacar en la Figura 2 y también en la 3, corresponde a que la adopción de clubes de fútbol profesional se ha mantenido constante en el tiempo, con un punto de inflexión recién hacia los años noventa y como las ciudades más lejanas del foco emisor se han 
ido incorporando a la actividad futbolística más tardíamente, lo anterior expresado principalmente por la lejanía geográfica al foco emisor. Sin embargo, si bien la distancia cumplió un papel fundamental en el patrón de distribución de este deporte, también lo fue la falta de "aficionados" y apoyo social que lograran sustentar la actividad. Lo anterior se observa al comprobar que hacia 1970 el fútbol profesional estaba comprendido entre las ciudades de Chillán y La Serena (ver Figura 1 para localización de los centros). Pero será en dicho periodo, que existirá una "deslocalización" de clubes de fútbol localizados en Santiago hacia las provincias como fueron los casos de Green Cross hacia la ciudad de Temuco y el de Iberia a Los Ángeles (1969). El proceso anterior fue potenciado por la creación de la Segunda División que originaba una mayor existencia de clubes y por tanto, una "distribución" de los aficionados y apoyo económico hacia los proyectos de provincia (SANTA CRUZ, 1991).

Otro aspecto paralelo a este proceso, será la función de las corporaciones industriales. En efecto, Huachipato (Concepción) tuvo el patrocinio y apoyo económico de la Compañía de Acero del Pacífico (CAP). También la Corporación Nacional del Cobre de Chile (CODELCO) tuvo un papel significativo al promover la fundación del Club Cobreloa en 1977 (Calama) y Cobresal hacia 1979 (Salvador). Otras industrias que apoyaron la creación de equipos son Bata (Deportes Melipilla), Super Pollo (Súper Lo Miranda) y la Empresa Portuaria de Chile (EMPORCHI), fomentando el nacimiento de Deportes Arica y Deportes Antofagasta.

Lo anterior no es sorprendente al observar lo ocurrido en otras latitudes referentes al proceso. En efecto, según Bale (1978), el grado de industrialización de las ciudades fue determinante para la adopción del profesionalismo en Inglaterra entre 1880 y 1900. Dicho proceso se "desarrolla" en Chile de manera más tardía, lo cual explica la diferencia del periodo con el caso inglés.

Otro aspecto interesante fue el proceso de regionalización vivido en el País hacia 1974, que configurara el interés de determinadas regiones por contar con fútbol profesional y también como una estrategia de incorporar al balompié nacional al sur de Chile, que hacia esos años estaba ausente. Así las ciudades de Puerto Montt, Valdivia y Osorno, capitales provinciales de la región de Los Lagos, fueron "invitadas" por la Asociación al fútbol profesional, producto de la "solicitud" realizada por la dictadura militar, mediante la Dirección General de Deportes y Recreación (DIGEDER) (SANTA CRUZ, 1991; PUMARINO, 2015). Según Álvarez (2011), la invitación se extendió a las ciudades de La Unión y Castro, quienes resolvieron no aceptarla.

Finalmente, las instituciones que asimilaron la profesionalización del deporte en estudio desde 1984 hasta la actual temporada 2014/2015, son posibles de considerar como "rezagados" en el proceso de difusión, dentro de este grupo se encuentran doce escuadras (aspecto no despreciable), en la que destacan Universidad de Concepción, Deportes Melipilla y Deportivo Santa Rosa Barnechea (actualmente, Athletic Club Barnechea), pero cuyas posibilidades de adopción están netamente asociadas a méritos deportivos, al entregar el fútbol aficionado un cupo al profesional. 
FIGURA 3

Difusión espacial por proximidad geográfica.

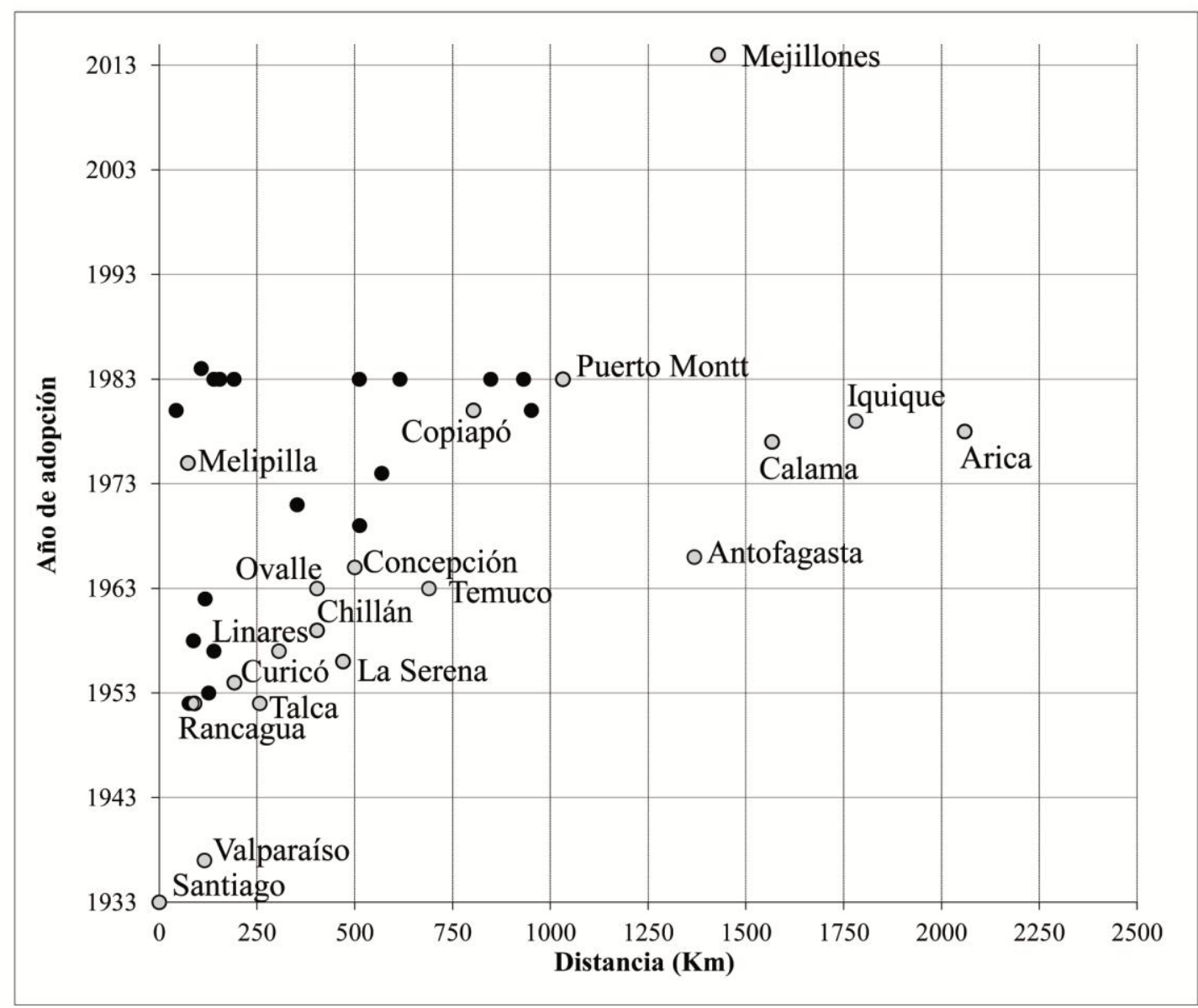

Fuente: elaboración propia.

Otros dos aspectos a destacar son observables en las Figuras 3 y 4 . El primero en relación a que, al igual que la concentración de población en el País, las ciudades adoptantes se concentran en su mayoría en las zona centro y sur ${ }^{5}$. En efecto, la distribución espacial indica que 15 urbes, es decir el $42 \%$ del total, se localizan a menos de $260 \mathrm{~km}$ de la Capital del País (foco emisor) y 11 a menos de $700 \mathrm{~km}$. Por lo tanto, prácticamente el $72 \%$ de las ciudades que integran el profesionalismo se encuentran a dicha distancia, que componen las regiones donde se concentra gran parte de la población del País. Las 10 restantes se localizan entre 700 y 2.000 $\mathrm{km}$. Esta característica denota que existe, sin duda, un vínculo entre la concentración de población en el País y la presencia de fútbol profesional.

El segundo aspecto a destacar está vinculado a que no necesariamente las ciudades con mayor población e importancia administrativa y funcional en el sistema urbano captaron primero la actividad. Esta característica es relevante, puesto que en un primer momento la proximidad parece haber jugado un papel fundamental en la difusión espacial del fútbol, ya que en un inicio arriva profesionalmente a Santiago, Valparaíso, Los Andes, Rancagua y Quillota. Pero en términos de su consolidación y persistencia, la presencia de población fue determinante. Además, si se considera lo expresado en las Figuras 4 y 5, por la juventud en la consolidación del sistema urbano chileno ${ }^{6}$, las ciudades cuando captaron la actividad no presentaban necesariamente una población de importancia (con excepción de Santiago, Valparaíso,

\footnotetext{
${ }^{5}$ Lo que corresponde a las regiones de Valparaíso, Metropolitana, O’Higgins, Maule y Araucanía.

${ }^{6}$ Recién en 1914 se articuló un tren entre Santiago y Puerto Montt. La región de La Araucanía fue "anexada" al territorio nacional hacia 1880.
} 
Concepción y Valdivia, quienes superaban los 100.000 habitantes). Mas bien la condición era la inexistencia de dicho deporte en el espacio.

FIGURA 4

Difusión espacial, según jerarquía urbana.

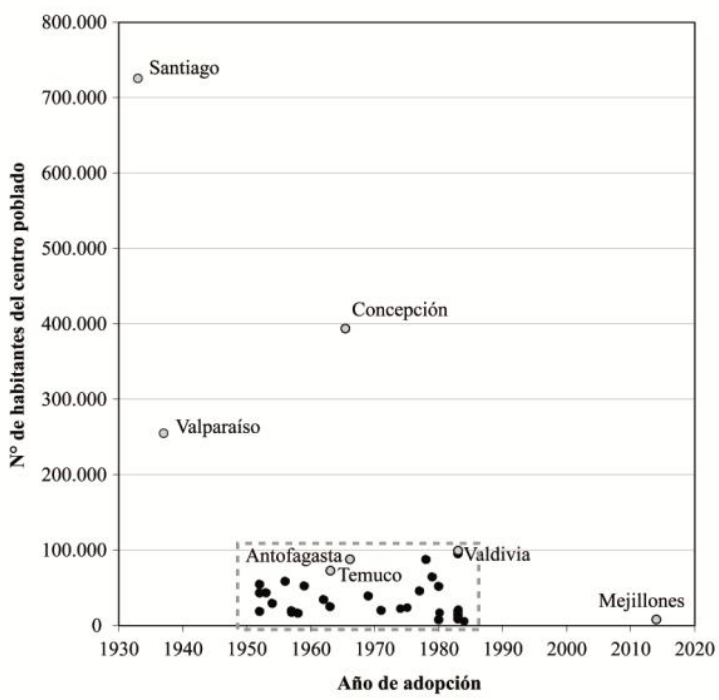

Fuente: elaboración propia.

FigURA 5

Difusión espacial, según jerarquía urbana (Ampliación)

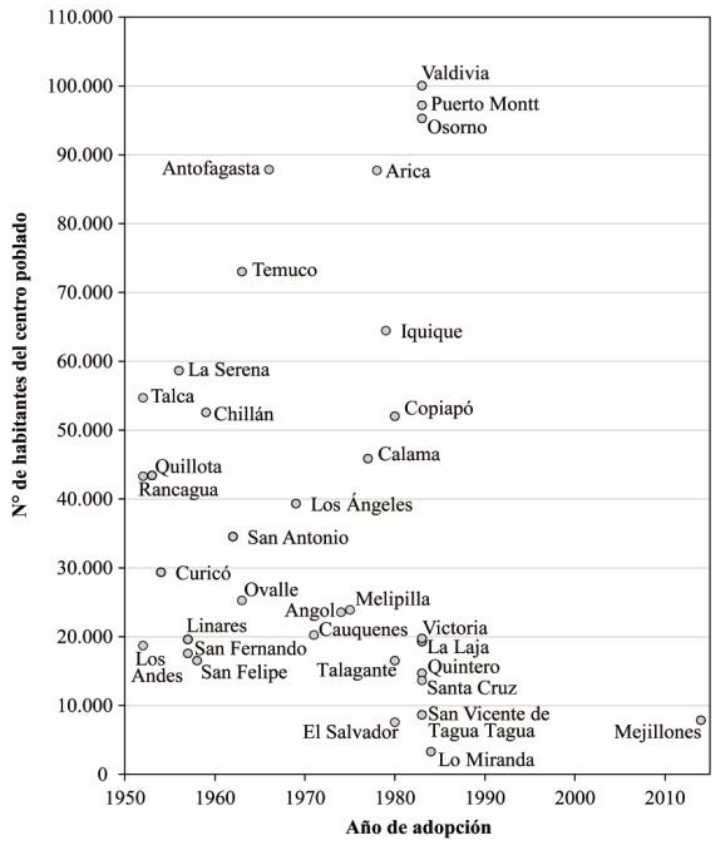

Fuente: Elaboración propia.

Otro elemento interesante a debatir, es la tardía incorporación de la ciudad de Concepción con algún club de fútbol profesional, lo cual solo sucederá hacia los años sesenta gracias a la siderúrgica ya señalada. En este punto es necesario destacar otro aspecto. En variadas ocasiones la innovación no fue posible de ser adquirida puesto que el organismo rector de la época, en este caso la "Asociación Central de Fútbol" (ACF) actuaba como una barrera a la propagación al impedir la incorporación al fútbol profesional. Fue el caso del club "Deportes Concepción" el 
cuál luego de tres peticiones es aceptado finalmente hacia 1966, algo discrecional si se piensa que ya había sido aceptado Huachipato y también llamativo al comparar con el caso de Dep. Temuco que pese a encontrarse más lejano ya había sido aceptado 3 años antes. Para el caso de Talca tal situación fue similar. Hacia 1933 pretendió ser parte del balompié profesional, pero su incorporación fue rechazada hasta 1952. Lo mismo ocurrió con Calama el año 1962, el equipo Sport Cóndor que contaba con el apoyo de Codelco (gracias a que el presidente del club, Carlos Seguel, era también el gerente de negocios de la estatal), inscribió su postulación para ingresar al profesionalismo. La respuesta a la solicitud fue negativa, por la lejanía de Calama con la Capital.

Si se realiza una comparación entre las ciudades que fueron rechazadas inicialmente para formar parte del fútbol profesional y las urbes que la ACF incitaron a unirse al profesionalismo, se observa que el argumento principal de la negativa fue la lejanía geográfica con Santiago (de donde provienen la mayoría de los participantes), y no se consideró la cantidad de población que componía cada centro urbano o las garantías en infraestructura ofrecidas por el club. Lo anterior se contrapone al caso de las ciudades sureñas, cuya invitación realizada tuvo como finalidad expandir el límite geográfico del balompié profesional en Chile, que a la fecha sólo llegaba hasta Temuco hacia los años sesenta. Con el paso de los años, la ACF le restó importancia al factor distancia y comenzó a considerar el componente infraestructura, en efecto, hacia 1963, cuando la ciudad de Temuco solicitó su ingreso al profesionalismo, a pesar de localizarse a casi $700 \mathrm{~km}$ de la Capital y de competir por el único cupo disponible contra equipos santiaguinos, vio aceptada su solicitud en el primer intento (PUMARINO, 2014).

Lo anterior denota dos aspectos claves de como se desarrolló el proceso de difusión. Por un lado existieron barreras institucionales que impedían la propagación, asociado a una lógica discrecional. En efecto, la actividad futbolista no así como otro tipo de innovaciones (enfermedades u otras) tendió a verse enfrentada a canales de transmisión con fuertes barreras donde los intereses particulares, se sobrepusieron a aspectos funcionales de los centros urbanos. Tal situación ocurrirá hasta 1981, cuando empezó a actuar el mecanismo de ascenso del fútbol aficionado al profesional, por lo que esta institución pierde poder toda vez que por méritos "deportivos" los clubes podrán ingresar al profesionalismo.

Por otro lado, en contraposición al primer aspecto, un segundo elemento resulta determinante: la distancia geográfica. Esta en cierta manera cumple la ley de que a mayor sea esta desde el foco emisor, menor será la capacidad de captación, situación que con las mejoras de transporte, reducción de costos, accesibilidad y comunicación tendió a acotarse sumado a la desaparición de las barreras institucionales. No obstante, la curva de adopción se mostró bastante establecida y creciente en el tiempo, lo anterior expresado principalmente por la proximidad en que ocurrían las captaciones en una primera etapa, para posteriormente los lugares más alejados ser incorporados, lo cual descartaría a la jerarquía como un elemento de importancia en la captación de la innovación.

\section{CONCLUSIONES}

El fútbol profesional en Chile se ha difundido siguiendo un patrón de distancia y cercanía al foco emisor, lo anterior supeditado por el poder institucional reflejado en la ACF, el ente que decidía qué equipos (ciudades) ingresaban al balompié profesional, hasta inicios de la década de los años ochenta.

Sin embargo, en la actualidad se han derribado las barreras institucionales que existían en el pasado y se fortalecieron los canales de transmisión, como lo son los medios de transporte y la accesibilidad de las ciudades, que eran el principal motivo de oposición de la ACF para permitir la llegada del fútbol profesional a los sectores más recónditos de Chile. Es por ello que no sorprende que el proceso de industrialización con sus mejoras en infraestructuras asociadas, haya fomentado el desarrollo de la actividad, que determinó en buena manera la expansión vivida por el fútbol en Chile. 
Como observación general, hasta la fecha, las ciudades más extremas que han adoptado el fútbol profesional son Arica y Puerto Montt, en los años 1978 y 1983, respectivamente, cubriendo $3089.42 \mathrm{~km}(53,1 \%)$ del territorio nacional. Hacía el norte ya no existen más ciudades a las que pueda llegar el profesionalismo (sólo algunos pueblos, como sería el caso de Putre). En el caso del sur, se observa que la difusión recién ha comenzado, ya que existen diversas urbes que aún no poseen esta innovación, como por ejemplo Puerto Varas, Calbuco, Llanquihue, Castro, Ancud (sectores en los que sí se ha adoptado el básquetbol profesional), La Unión, Chaitén, Coyhaique, Puerto Aysén, Punta Arenas, entre otras. Dicho de otra manera, existen $2730.35 \mathrm{~km}(46,9 \%)$ en el sector austral del País, donde el fútbol profesional todavía no ha sido acogido, lo cual sería posible atribuir a condiciones climáticas y culturas, pero sin duda otros estudios debería profundizar en la materia.

En términos del costo de desplazamiento, podría ser un motivo para explicar su inexisten a Coyhaique o a Punta Arenas, pero no solo su valor, sino que la gran mayoría de las conexiones obligan primero hacer escala en Santiago, por lo que para los equipos de fútbol profesional pertenecientes a urbes localizadas al sur de la Capital es un costo adicional de movilización tanto en recursos como en tiempo.

Cabe mencionar que queda descartada la influencia del tamaño de población en la difusión espacial del fútbol profesional en Chile, ya que no necesariamente las ciudades con mayor población y rol administrativo en el sistema urbano captaron primero la actividad. Esto se expresa a que la ACF no tomó en cuenta los aspectos funcionales de los centros urbanos que postulaban al profesionalismo. No obstante, es innegable el papel que ha jugado el factor poblaión de las ciudades al momento de captar y hacer perdurar la actividad, ya que esta se traduce en un mayor número de aficionados y un mayor apoyo social que permiten sustentar la llegada del fútbol profesional a una determinada urbe.

En cuanto a la adopción de este deporte en Chile, los grupos de adoptadores observados en la Figura 2 responden a momentos emblemáticos dentro del fútbol profesional chileno, como el ingreso del primer club provinciano al profesionalismo, la instauración de una Segunda División, el apoyo industrial a la actividad en ciertas urbes y la invitación de la Asociación Central de Fútbol a ciudades localizadas en el sector sur del País, debido a la orden recibida desde la DIGEDER.

\section{REFERENCIAS BIBLIOGRÁFICAS}

ÁLVAREZ, P. http://www.elvacanudo.cl/noticia/deporte/felices-28-anos-provincial-osorno. [Consulta: 7 de octubre 2014].

BALE, J. (1978). Geography. "Geographical diffusion and the adoption of professionalism in football in England and Wales", Vol. 63, n³, p. 188-197.

BALE, J. (1998). Revista Efdeportes. "La hinchada virtual; el fututo paisaje del fútbol", vol. 3, $\mathrm{n}^{\circ} 10$.

BOUKERZAZA, H. (2006). Insaniyat. "Géographie et hiérarchie du football en Algérie: l'exemple du Nord-Est Algérien". Vol. 10, n³ 34, p. 39-55.

BONNASSIOLLE, M. (2012): Fútbol obrero y popular: masificación, popularización y sociabilidad obrera en Chile, 1890-1930. Tesis para optar al grado de Licenciado en Historia con mención en estudios Culturales, Universidad Academia de Humanismo Cristiano, Santiago, Chile, 106 pp.

CAPEL, H. (2001). Revista Bibliográfica de Geografía y Ciencias Sociales. "Mascarenhas de Jesus, Gilmar. A bola nas redes e o enredo do lugar: uma geografia do futebol e de seu advento no Rio Grande do Sul”, Vol. 6, n³01.

CAPO, J.

http://www.uib.es/depart/deaweb/webpersonal/javiercapo/EcRegional/temas/crecimiento.pdf . [Consulta: 2 de marzo 2015].

CASSASUS, A. y GÓMEZ, G. (2012): Conocimiento y búsqueda de oportunidades de negocios en el fútbol amateur en la región Metropolitana. Tesis para optar al grado de Ingeniero 
Comercial, mención en Administración, Escuela de Economía y Administración, Universidad de Chile, Santiago, Chile, 36 pp.

DURÁN, G., GUAJARDO, M., MIRANDA, J., SAURE, D., WEINTRAUB, A., CARMASH, A. y CHAIGNEAU, F. (2005). Revista Ingeniería de Sistemas. "Programación Matemática Aplicada al Fixture de la Primera División del Fútbol Chileno", vol. XIX, p. 29-48

DRUT, B. (2014) : Économie du football professionnel, La Découverte Francia, Paris, 125 pp.

ESCOBAR, J. (2011): Estudio exploratorio de la relación entre el fútbol y la política a partir de las selecciones chilenas participantes en los mundiales de Alemania 1974 y España 1982. Tesis para optar al título profesional de Sociólogo, Escuela de Sociología, Universidad de Arte y Ciencias Sociales, Santiago, Chile, 270 pp.

FIFA. http://fr.fifa.com/classicfootball/history/the-game/origins.html. [Consulta: 13 de noviembre 2014].

FIGALLO, P., MUÑOZ, P. y SALHE, P. (2003): Culturas juveniles e identidad: El caso de las barras bravas del fútbol. Tesis para optar al grado de licenciado en Psicología, Facultad de Ciencias Humanas y Educación, Universidad Diego Portales, Santiago, Chile, 128 pp.

GROSJEAN, F. (2006). Mappemonde. "Processus de diffusion du football en Franche-Comté", vol. $1 \mathrm{n}^{\circ} 81$, p. $18-26$.

GUERRERO, B. (2006). Revista de Ciencias Sociales. "Fútbol en el norte grande de Chile: Identidad nacional e identidad regional", vol. 15, n 16, p. 4-15.

INSTITUTO NACIONAL DE ESTADÍSTICAS, INE. (1999): Población de los centros poblados de Chile 1875-1992. Departamento de Servicios al Usuario y Difusión, Santiago, $86 \mathrm{pp}$.

INSTITUTO NACIONAL DE ESTADÍSTICAS, INE. (2005): Censo ciudades, pueblos, aldeas y caseríos. Gobierno de Chile, Santiago, 300 pp.

INFANTE, R. (1980). Revista de Geografía Norte Grande, "Difusión y expansión urbana como procesos análogos", vol. 7, n 7, p. 57-61.

MARAMBIO, L. (2010): Valparaíso y Wanderers: Cosmopolitas y Pioneros. Memoria para optar al título de Periodista, Instituto de la Comunicación e Imagen, Universidad de Chile, Santiago, Chile, 183 pp.

MARÍN, E. (1995): Centenario: Historia total del fútbol chileno (1895-1995), EME Santiago, $355 \mathrm{pp}$.

MASCARENHAS DE JESUS, G. (1998). Revista Efdeportes. "Fútbol y modernidad en Brasil: La geografía histórica de una novedad", vol. 3, n 10.

MASCARENHAS DE JESUS, G. (2000). Revista Electrónica de Geografía y Ciencias Sociales. "Considerações teórico-metodológicas sobre a difusão do futebol", vol. 4, n 69.

MASCARENHAS DE JESUS, G. (2008). Scripta Nova: Revista Electrónica de Geografía y Ciencias Sociales. "Football, globalisation and local identity in Brazil. Esporte $e$ Sociedade", vol. $3, \mathrm{n}^{\circ} 8$.

MEYSSONNIER, F. y MINCHENEAU, M. (2013). Finance Contrôle Stratégie "Le contrôle de gestion des clubs de football professionnel", vol. 16, $\mathrm{n}^{\circ} 4$.

MINISTERIO DE OBRAS PÚBLICAS, MOP. (s.f.). http://servicios.vialidad.cl/Distancias/Distancias.asp. [Consulta: 25 de agosto 2014].

OMAÑA, J. (2007): El impacto del fútbol en la integración Latinoamericana. Tesis para optar al título en Licenciatura en Relaciones Internacionales, Departamento de Relaciones Internacionales y Ciencias Políticas, Universidad de las Américas Puebla, Puebla, México.

PACHÉ, G. y N'GOALA, P. (2011). Management \& Avenir. "Les stratégies de création et d'appropriation de la valeur dans un contexte d'incertitude majeure: le cas du football professionnel", vol. 6, $\mathrm{n}^{\circ} 46$, p. 53-78.

PARRA, A. (2007): Sociología del fútbol: Desarrollo de una pasión ecuménica. Si el mundo es redondo, hagámosle un gol al arcoiris. Tesis para optar al grado de Licenciado en Sociología, Escuela de Sociología, Universidad Central, Santiago Chile.

PICKENHAYN, J. (2001). Boletín de Gra, Buenos Aires, Sociedad Argentina de Estudios Geográficos Pickenhayn. "Difusión y dispersión en la historia de la geografía. Innovaciones, 
movimientos de masas y brotes epidémicos como campo de acción del geógrafo", $\mathrm{n}^{\circ}$ 119, $\mathrm{p}$. 37-61.

PUMAIN, D. y SAINT-JULIEN, T. (2001) : Les interactions spatiales, Armand Colin, Paris, $222 \mathrm{pp}$.

PUMARINO, F. http://www.futuro.cl/2014/04/el-deportes-temuco-original-futbol-con-lluvia/. [Consulta: 17 de octubre 2014].

PUMARINO, F. http://www.futuro.cl/2015/02/valdivia-osorno-y-puerto-montt-la-ultimaexpansion-del-futbol/. [Consulta: 13 de marzo 2015].

RAVENEL, L. (1997): Le football de haut niveau en France: Espaces et territoires. (Tesis de doctorado en Estructuras y Dinámicas Espaciales), Académie D'Aix-Marseille, Université D'Avignon et des Pays de Vaucluse, Avignon, Francia.

RAVENEL, L. (1998). Espace Géographique. "Hiérarchies urbaines, hiérarchies sportives: quand le football français s'écarte de la norme européenne", vol. 27, nº 4, p. 339-348.

REC. SPORT SOCCER STATISTICS FOUNDATION, RSSSF. (s.f.). http://www.rsssf.com/. [Consulta: 25 de enero 2014].

SANTA CRUZ, E (1991): Crónica de un encuentro: Fútbol y cultura popular, Instituto Profesional Arcos Santiago, $159 \mathrm{pp}$.

SCHOTTE, M. (2014). Revue Française de Socio-Économie. "La structuration du football professionnel européen Les fondements sociaux de la prévalence de la spécificité sportive", vol. $1, \mathrm{n}^{\circ} 13$, p. $85-106$.

THERY, H. (2006). Mappemonde. "Futebol et hiérarchies urbaines au Bresil", vol. 1, n 81, p. 27-38.

TULLA, A. (1983). Documents d'Anàlisi Geogràfica. "El modelo de difusión de T. Hagerstrand. Una aplicación a la ganadería del Pirineo Catalán”, vol. 2, n 2, p. 69-160.

VALENZUELA, E. y VERGARA, C. (2013). . Revista de Ciencias Sociales. "Hacía los imaginarios urbanos de Valparaíso a través del fútbol: El caso de Santiago Wanderers", vol. $21, \mathrm{n}^{\circ} 31$, p. $138-157$.

VILLENA, S. (2003). Gol-balización, identidades nacionales y fútbol. En Alabarces, P. (Ed.). Futbologías: Futbol, identidad y violencia en América Latina. CLACSO, Buenos Aires, pp. 257-271. 